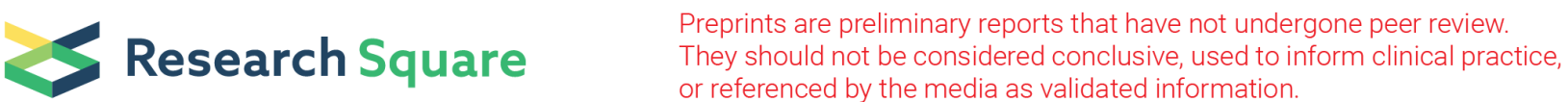

\section{Silibinin Promotes Bone Regeneration Through HIF- 1a/VEGF and Notch Signaling Pathway in Ovariectomized Rats}

\section{Zhoushan Tao ( $\nabla$ tzs19900327@126.com )}

The First Affiliated Hospital of Wannan Medical College: Yijishan Hospital of Wannan Medical College

Tian-Lin Li

The First Affiliated Hospital of Wannan Medical College: Yijishan Hospital of Wannan Medical College

Min Yang

The First Affiliated Hospital of Wannan Medical College: Yijishan Hospital of Wannan Medical College Hong-Guang Xu

The First Affiliated Hospital of Wannan Medical College: Yijishan Hospital of Wannan Medical College

\section{Research}

Keywords: Postmenopausal osteoporosis, Bone defect, Silibinin, Bone regeneration.

Posted Date: June 15th, 2021

DOI: https://doi.org/10.21203/rs.3.rs-618566/v1

License: (c) (i) This work is licensed under a Creative Commons Attribution 4.0 International License. Read Full License 


\section{Abstract \\ Objective}

The purpose was to observe whether systemic administration with silibinin(SIL) have an positive effect on bone defect regeneration through HIF-1a/VEGF and Notch signaling pathway in an ovariectomized(OVX) rat model.

\section{Methods}

The MC3T3-E1 cells were co-cultured with lower SIL and higher SIL and induced to osteogenesis, and the cell viability, osteogenic activity were observed by Cell Count Kit-8(CCK-8), Alkaline phosphatase (ALP) staining, Alizarin Red(RES) staining and Western blotting(WB). After the drilling defect model was established, the OVX rats were treated with SIL for 12 weeks. Micro-CT, histology and Reverse transcription-quantitative polymerase chain reaction (RT-qPCR) analysis were used to observe the therapeutic effect and explore the possible mechanism.

\section{Results}

CCK-8, ALP and ARS staining results show that the cell mineralization and osteogenic activity of LSIL and HSIL group is significantly higher than the Con group. Protein expressions show that related regulatory proteins such as ALP, OPN, RUNX-2, OC, VEGFA, HIF-1a, Notch 1, JAG 1, HEY 1 and HES 1 of LSIL and HSIL group are significantly higher than Con group. Micro-CT and Histological analysis evaluation show that group SIL + OVX presented the stronger effect on bone regeneration, bone mineralization, higher expression of VEGFA and HIF-1a, when compared with OVX group. RT-qPCR analysis shows that SIL + OVX group showed increased Notch 1, HES1, HEY1 and JAG1 than the OVX group $(p<0.05)$.

\section{Conclusions}

Our current study demonstrated that systemic administration with SIL is a scheme for rapid repair of femoral condylar defects, and these effects may be achieved by activating HIF-1a/VEGF and Notch signaling pathway.

\section{Introduction}

Postmenopausal osteoporosis is characterized by reduced bone mineral density (BMD) and deterioration of bone microarchitecture resulting in increased bone fragility and an increased risk of developing fractures, which commonly happened in postmenopausal women[1, 2]. Most osteoporotic fractures and bone defects are commonly seen and happen after low-energy trauma[3,4]. The bone is a kind of mineralized connective tissue which has the ability to repair minor injuries by remodeling. When the bone 
defect reaches a certain limit, it is difficult to complete the defect healing by its own repair ability. The autologous bone graft has good biocompatibility and bone-inducing ability, demonstrating the gold standard for bone defect healing[5]. However, autologous bone grating is limited by donor site morbidity, which will inevitably lead to extension of surgical time, increase of surgical sites and infection chance and aggravation of patient pain[6, 7]. Especially for the elderly with many basic diseases and they are not allowed to tolerate long-term surgery. Therefore, it poses new challenges for our clinicians.

Silibinin(SIL), a flavonoid obtained from the dried fruits of Silybum marianum L. [8], is mainly composed of four flavonoids and can exert multiple biological effects[9]. Previous studies also have reported that SIL supplementation is not only able to prevent oxidative stress-induced cell cytotoxicity in bone marrow mesenchymal stromal cells (MSCs), but also efficiently inhibited oxidative stress and protects their osteoblastic differentiation[10-13]. Currently, SIL is also reported to be potential in regulating osteoblastic cell growth and functions, and maintaining bone mass[14, 15]. Vascular endothelial factor (VEGF) can not only promote survival, proliferation and migration of endothelial cells, but also stimulate the growth of new blood vessels into damaged sites and regulate osteogenic growth factors to stimulate bone repair and bone regeneration.[16]. Hypoxia inducible factor 1(HIF-1), a transcription factor, expressed ubiquitously in almost all mammalian cells, which becomes activated under hypoxia[17]. Moreover, VEGF is a major target gene of HIF-1a. Activation of VEGF signaling activates the HIF-1a signaling pathway to promote angiogenesis and bone growth[18]. Recently, accumulating evidence proves that notch signaling regulates mesenchymal stem cells proliferation and differentiation, skeletal tissue development and regeneration[19].

Although current studies have confirmed that silibinin play an important role in the process of bone remodeling, systemic administration of silibinin in the treatment of osteoporotic bone defects is lacking and limited. Based on these previous studies, hypothesized that systemic administration with silibinin may have a positive effect on bone defect regeneration through Notch signaling pathway and HIF$1 a / V E G F$ signaling pathway in an OVX rat model. The aim of the present study was to investigate the effect of systemic administration with silibinin on bone defect in an OVX rat model, and preliminary exploration of possible mechanisms by activating the Notch signaling pathway and HIF-1a/VEGF signaling pathway.

\section{Materials And Methods}

\section{Experimental animals}

30 mature female Sprague Dawley rats(Purchased at Zhejiang Laboratory Animal Center, Zhejiang, China), aged 3 months with a body weight of $230 \pm 26 \mathrm{~g}$, were included in this study. Every four animals were acclimatized in a standard cage under a 12-hour light-dark cycle and allowed free access to water and rodent diet. All the animal experiments were conducted in accordance with international standards on animal welfare as well as being compliant with the Animal Research Committee of the university(approval number:LLSC-2020-082). 


\section{Animal experiments}

All osteoporosis models of estrogen deficiency induced by bilateral ovariectomy were established as described previously[20, 21]. 12 weeks after sham operation(Sham) and bilateral ovariectomy(OVX), all rats were randomly divided into 3 groups: Sham group (Sham), OVX rats group (OVX) and OVX rats + silybin treatment group (SIL+OVX). Next, the rats of three groups established an intramedullary defect in the distal femur ( $1.5 \mathrm{~mm}$ in diameter $\times 4 \mathrm{~mm}$ in depth) using a $1.5 \mathrm{~mm}$ diameter clinical drill bits(Stryker Corporation, US) as described previously[22, 23]. The rats were classified to SIL+OVX were treated with silibinin intraperitoneally with a dose of $50 \mathrm{mg} / \mathrm{kg}$ (Sigma-Aldrich)[24]. Two intraperitoneal injections of calcein $(20 \mathrm{mg} / \mathrm{kg})$ were injected on the 3rd and 10th day before the rats were sacrificed. After 12 weeks of treatment, the rats undergoing bone defect surgery were sacrificed using an overdose of chloral hydrate. Serum and femur samples were harvested. Femurs were fixed at $4^{\circ} \mathrm{C}$ with $4 \%$ paraformaldehyde. Whole blood was frozen at $-80^{\circ} \mathrm{C}$ for later use.

\section{Cell culture and cell viability Measurement}

As an osteoblast precursor cell line, MC3TE-E1 was obtained from the Institute of Biochemistry and Cell Biology, CAS (Shanghai, China). MC3TE-E1 were cultured in 24-well plates at $1 \times 10^{4}$ cells per well with growth culture medium. After culturing for $24 \mathrm{~h}$, cells were treated with phosphate-buffered saline (PBS), silibinin $(20 \mu \mathrm{mol} / \mathrm{l}, \mathrm{LSIL})$ and silibinin $(50 \mu \mathrm{mol} / \mathrm{l}, \mathrm{HSIL})$. The Cell Count Kit-8 (YEASEN, Shanghai, China) was used to evaluate the effects of silibinin on cell viability.

\section{Alkaline phosphatase (ALP) staining and Alizarin Red(RES) staining}

MC3TE-E1 cells were plated at a density of $1 \times 10^{4}$ cells $/ \mathrm{ml}$ in 24 well plates and cultured in growth medium supplemented with $10^{-8} \mathrm{M}$ dexamethasone (Sigma), $50 \mu \mathrm{g} / \mathrm{ml}$ ascorbic acid (Sigma) and $5 \mathrm{mM}$ $\beta$-glycerol phosphate (Sigma). Then the medium was added with phosphate-buffered saline (PBS), silibinin $(20 \mu \mathrm{mol} / \mathrm{I}, \mathrm{LSIL})$ and silibinin $(50 \mu \mathrm{mol} / \mathrm{l}, \mathrm{HSIL})$. The medium was changed every four days during osteogenic differentiation. After induction for 14 and 21 days, osteogenesis was evaluated by staining MC3T3-E1 osteoblasts with ALP substrate mixture (ALP staining kit, Sigma) and Alizarin Red reagent (RES, Cyagen Biosciences, Guangzhou, China) as protocol described, respectively.

\section{Microcomputed tomography (Micro-CT) scanning}

The distal femur was analyzed with anisotropic voxel size of 10 $\mu \mathrm{m}$ through the Micro-CT (Bruker Skyscan 1272 system, Kontich, Belgium). The parameter is set to $55 \mathrm{kV}$ and $114 \mathrm{~m} \mathrm{~A}$ with a thickness of $0.048 \mathrm{~mm}$ per slice in medium-resolution mode, 1024 reconstruction matrix, and $200 \mathrm{~ms}$ integration time. These images and parameters of trabecular bone parameters with a distance of $1 \mathrm{~mm}$ proximal from the end of the growth plate in femoral metaphysis were compared between the Sham group and OVX group to confirm the osteoporosis rat model. For evaluation of bone formation in the defect area, the central 1.5$\mathrm{mm}$-diameter region of the defect was defined by drawing circular contour as a consistent volume of interest (VOI). After 3D reconstruction, bone mineral density(BMD), bone mineral content(BMC), bone 
volume fraction(BV/TV), trabecular number(Tb.N), trabecular thickness(Tb.Th), trabecular separation(Tb.Sp) were automatically determined for identification of osteoporosis model while BMD, BV/TV, Tb. N, Tb. Th, Tb. Sp, the mean connective density (Conn.D) in VOI regions were used to evaluate new bone formation, using a protocol provided by the manufacturer of the Micro-CT scanner as previously described[25, 26].

\section{Histomorphometric analysis and immunofluorescence staining}

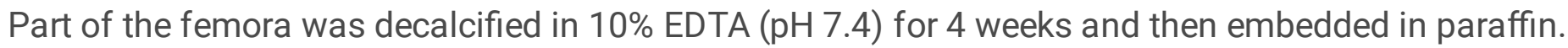
Four-micrometer-thick longitudinally oriented along the defect sections were used for staining. HE staining was performed to observe the trabecular bone and bone formation in the defect area. VEGF and HIF-1a staining were used to quantify the expression of angiogenic factors in the defect area. In brief, fresh bone sections were stained with individual primary antibodies to rats VEGFA(Abcam, ab206887, 1:100) and HIF-1a(Abcam, ab180880,1:100), overnight at $4^{\circ} \mathrm{C}$. Subsequently, the secondary antibodies conjugated with fluorescence (Jackson Immuno Research, 415-605-166, 1:500; 315-605-003, 1:250) were used at room temperature for $1 \mathrm{~h}$ while avoiding light and observed under a confocal microscope (FLUOVIEW FV300, Olympus). Calcein double labelling in undecalcified bone slices were observed under a fluorescence microscope(FLUOVIEW FV300, Olympus) to quantify bone mineralization in the defect area.

\section{Western blot analysis}

Cell extracts of bone tissue in the defect area after 4 weeks of EDTA decalcification or MC3TE-E1 cells 3 days after drug intervention were prepared for Western blotting as previously described. Then, $20 \mu \mathrm{g}$ of total cell lysate protein was prepared in Pro-PREPTM Protein Extraction Solution (Boca Scientific Inc., Boca Raton, $\mathrm{FL}$ ) and electrophoresed. The primary antibodies against the following proteins: alkaline phosphatase (ALP, Abcam, ab198554, 1:1000), RUNX family transcription factor 2(RUNX-2, Abcam, ab236639, 1:1000), osteopontin(OPN, Abcam, ab214050, 1:1000), Osteocalcin(OC, Abcam, ab133612, 1:1000), Anti-Notch1 (Abcam,ab52627,1:1000), Anti-HEY1 (Abcam,ab154077,1:1000), Anti-Jag1 (Abcam,ab109536,1:1000), Anti-HES1(Abcam, ab119776,1:1000), VEGFA(Abcam, ab214424, 1:1000) and HIF-1a(Abcam, ab180880, 1:1000). Protein expression levels were normalised to Glyceraldehyde 3 phosphate dehydrogenase (GAPDH; Boster, Wuhan, China, 1:2000) protein levels. HRP-conjugated goat anti-rabbit (Santa Cruz Biotechnology, Santa Cruz, CA) were used as secondary antibodies. Blots were imaged using an iBrightCL1000 (Invitrogen, Carlsbad, CA).

\section{Reverse transcription and real time polymerase chain reaction (RT-PCR) analysis}

According to the manufacturer's instructions, Total messenger RNA (mRNA) was extracted using the total RNA extraction kit (Takara, Kusatsu, Japan). Complementary DNA (cDNA) was obtained from total RNA using first Strand cDNA Synthesis Kit (Toyobo, Ōsaka, Japan). Then synthetic cDNAs and specific primers were used for qRT PCR with the TB Green ${ }^{\text {TM }}$ Premix Ex Taq II (Tli RNaseH Plus) kit (Takara, Kusatsu, Japan) on the CFX Connect ${ }^{\mathrm{TM}}$ Real-Time System (Bio-Rad, Singapore). GAPDH was used as an 
internal control. Sequences of primers for the reference gene (GAPDH) and interested genes are listed in table 1.

Table 1 Nucleotide sequences for real-time RT-PCR primers.

\begin{tabular}{|llll|}
\hline Genes & Forward (5'-3') & Reverse $\left(5^{\prime}\right.$-3') & $\begin{array}{l}\text { Product } \\
\text { length }\end{array}$ \\
Notch 1 & CGGGGCTAACAAAGATATGC & CACCTTGGCGGTCTCGTA & 68 \\
HES1 & GGAAATGACAGTGAAGCACCT & CAGCACACTTGGGTCTGTG & 78 \\
HEY1 & GGCAGGAGGGAAAGGTTACT & CTCAGATAACGCGCAACTTC & 79 \\
JAG1 & GGCAACACCTTCAACCTCA & GCCTCCACAAGCAACGTATAG & 103 \\
GAPDH & TGCGATGGGTGTGAACCACGAGAA & GAGCCCTTCCACAATGCCAAAGTT & 130 \\
\hline
\end{tabular}

\section{Statistical analysis}

Data were expressed as Mean \pm standard deviation. Statistical significances among three groups were determined by one-way analysis of variance and the Student $s$ t-test. The value of $p<0.05$ was considered statistically significant.

\section{Result}

\section{Osteoporosis animal model validation}

A total of 5 rats died during the experiment, including anesthetic accidents, infection and surgical accidents. No animal death was found in the first operation. The death of rats occurred during or after the second operation, including Sham group $(n=1)$, OVX group $(n=2)$ and SIL+OVX group $(n=2)$. After 12 weeks of ovariectomy and sham operation, the femurs of 5 rats were randomly selected and observed by Micro-CT. The BMD of metaphysis of the femur in the OVX group $\left(164.30 \pm 26.43 \mathrm{mg} / \mathrm{cm}^{2}\right)$ are significant statistical differences between Sham group $\left(233.32 \pm 31.23 \mathrm{mg} / \mathrm{cm}^{2}, \mathrm{P}<0.05\right)$. These results indicate that the osteoporotic rat model induced by ovariectomized surgery in our experiment achieves the expectation.

\section{Micro-CT evaluation}

The 2D scan images(Figure 1. A-C) and 3D reconstruction images(Figure1. a-c) of Micro-CT clearly shows us the bone remodeling of the defect area after 12 weeks of treatment with different intervention methods. As we expected, the defect area of the Sham group was almost filled with bone tissue, while large amounts of bone tissue was found in the SIL+OVX group, but it was difficult to find the bone tissue in the OVX group. The quantitative results were expressed as BMD, BV/TV, Tb. Th, Tb. N, Conn. D and Tb. $\mathrm{Sp}$ (Figure $1 \mathrm{D}$ ). Therapy with SIL showed positive effects on all micro-CT parameters. Compared to 
groups OVX, SIL treatment shows the better bone microscopic parameters including the higher BMD, BV/TV, Tb. N, Conn.D, Tb. Th, and the lowest Tb. Sp $(P<0.05)$.

\section{Histological and Fluorescent analysis}

Histological and fluorescent images showing bone repair in defect for different treatments, as shown in Figure 2. In 12 weeks, a large amount of bone tissue fills the defect area in the Sham group. In the OVX group, only a very small amount of new bone tissue can be observed; and large defect areas still exist, while the defect area of SIL+OVX group was significantly reduced in rats. In fluorescent analysis, treatment with SIL showed the larger calcein green-marked defect area $(p<0.05)$, and exhibited the higher values of relative bone mineralization(green/green marked defect area) $(p<0.05)$, compared to that of the OVX group.

The angiogenesis regulator of bone defect measured by immunofluorescence and WB clearly show us the expression of HIF-1a and VEGFA of the defect area after 12 weeks of treatment with different intervention methods(Figure 2). As we expected, the defect area of the Sham group was almost filled with immunofluorescence for HIF-1a and VEGFA, while large amounts of immunofluorescence was found in the SIL+OVX group, but it was difficult to find immunofluorescence in the OVX group. The quantitative results measured by WB were expressed as HIF-1a and VEGFA. Therapy with SIL showed positive effects on HIF-1 $a$ and VEGFA expressions. Compared to groups OVX, SIL treatment shows the higher protein expression with HIF-1 $a$ and VEGFA $(P<0.05)$.

\section{RT-qPCR analysis}

Gene expression of defect area bone tissue after different treatment, as shown in Figure 3. In 12 weeks, $\mathrm{SIL}+\mathrm{OVX}$ group showed increased Notch $1, \mathrm{HES} 1, \mathrm{HEY} 1$ and JAG1 than the OVX group $(\mathrm{p}<0.05)$. These results indicate that the Notch pathway of SIL treatment is activated, and the expression of Notch 1 , HES1, HEY1 and JAG1 is up-regulated.

\section{Cell viability and function and related protein expression}

In order to determine the effect of silibinin on MC3T3-E1 cells viability and function and related protein expression, this study further conducted Cell Count Kit-8, ALP staining, RES staining and WB analysis. As shown in Figure $4 \mathrm{~A}$, the cell viability of HSIL and LSIL group were significantly higher than that of Con group $(P<0.05)$. ALP staining, RES staining with quantification of area in osteogenic differentiation of MC3T3-E1 cells is shown in Figure 4D. The mineralized nodules (number per well), mineralized area (\%), ALP activity and ALP gray value of HSIL and LSIL group were significantly higher than that of Con group $(P<0.05)$. The protein expressions including ALP, OPN, RUNX-2, OC, VEGFA, HIF-1a, Notch 1, JAG 1, HEY 1 and HES 1 of HSIL and LSIL group were significantly higher than that of Con group $(P<0.05)$. These results indicate that the treatment with silibinin can significantly increase MC3T3-E1 cells viability and function and related protein expression by activating Notch signaling pathway and HIF-1a/VEGF signaling pathway. 


\section{Discussion}

So far, studies have confirmed that osteoporotic bones undergo a prolonged and impaired healing process in clinical postmenopausal osteoporotic women and experimental estrogen deficiency animal model[27, 23]. Despite substantial advances in the field of bone repair, strengthening the ability of bone formation remains a major challenge that has held back the clinical use of artificial bone biomaterials. In the present study, we report that systemic administration with silibinin could promote bone regeneration in OVX rats. Our results show that the successful regeneration of bone defect was achieved based on evaluation of Micro-CT, and also confirmed with histological evaluation.

In this study, we compared the changes of bone mineral density and bone mass between Sham-operated rats and OVX rats, and successfully established an osteoporotic rat model through detection and evaluation of bone mineral density. In this model, a standard perforation $1.5 \mathrm{~mm}$ in diameter was generated at femoral condyle. Histological evidence and Micro-CT 3D reconstruction image demonstrated that very little new bone tissue was observed within the defected zone following drill-hole surgery in OVX group. The bone defect model used in this study is a mature and easily repeatable model originally used to intramem-branous bone formation, which is better than previously reported using an internal or external fixation model[28]. Micro-CT reconstruction and histological examination consistently demonstrated less new bone formation in defect region in OVX rats, suggesting that bone repair ability was impaired during bone healing in the OVX-induced osteoporotic bone. In the study, more new bone tissue in the defect area was observed in the SIL + OVX group at 12 weeks than in the OVX group, suggesting that systemic administration with silibinin could accelerate the healing response. Accordingly, the results of this experiment suggest that systemic administration with silibinin may hold promise for improving bone defect repair under osteoporosis.

In order to further investigate the potential mechanisms, we also performed bone tissue immunofluorescence, qRT-PCR and western blotting experiments to analyze the mRNA expression of related gene, regulatory factor and protein contents. Previous studies indicated that signaling pathway also plays vital roles in mineralization of bone tissue via a direct regulation effect on osteoblastic activity[29, 30]. Therefore, RT-PCR was conducted to detect the expression levels of several Notch target genes for bone tissue in the defect area of rats in each group. The results showed that the ovariectomy induces estrogen deficiency decreased the expression of target genes in bone tissues including Notch 1 , HES1, HEY1 and JAG1. What's more, the decreased signaling pathway by ovariectomy was restored by SIL treatment. Recently, a large body and animal of emerging evidence has proved that angiogenesis plays a key role in bone repair[31,32]. Previous reports reached consensus that the tightly coupled angiogenesis and osteogenesis play an important role in bone regeneration[33]. Similarly, angiogenesis is regulated by a variety of growth factors, such as VEGFA and HIF-1a[34-36]. Among them, VEGFA and HIF-1a are the two most important factors that seem to play coordinated roles in vascular development to support bone regeneration. Osteoblast experiments found that treatment with silibinin can significantly increase MC3T3-E1 cells viability and function and related protein expression by activating Notch signaling pathway and HIF-1a/VEGF signaling pathway. Combining the above results, what we can 
explain is: systemic administration with Sil can markedly promote osteogenesis and angiogenesis, which results in a systemic environment that is more conducive to bone repair and ultimately accelerates bone formation.

As far as we know, this is the first study of the effect of systemic administration with SIL on the regeneration of bone defect under osteoporotic conditions. Nevertheless, this study had several deficiencies. Firstly, the mechanisms underlying the effects of SIL on osteogenic differentiation of MSCs and osteoclasts should be elucidated. In vitro experiments of osteoblasts lack pathway agonists and inhibitors. Moreover, local delivery systems that allow the sustained release of SIL should be developed for effective bone regeneration in vivo. In addition, the optimal dosage of SIL should be determined for bone regeneration by using animal studies. Besides, there is no positive control group for animal experiments.

\section{Conclusion}

In summary, our study suggests that treatment with SIL may be a useful method to improve the initial bone regeneration of defects by increasing bone formation and angiogenesis in osteoporotic rats. Besides, this benefit effect may be mediated by HIF-1a/VEGF and Notch signaling pathway.

\section{Declarations}

\section{Acknowledgments}

We are very grateful to Hong-Guang Xu and Min Yang for providing the assistance in the experimental design stage.

\section{Authors' contributions}

Zhou-Shan Tao, Tian-Lin Li, Hong-Guang Xu and Min Yang performed the research design and the paper drafting. Zhou-Shan Tao performed the experimental guidance and manuscript revision. Tian-Lin Li performed the collection and analysis of experimental data. Hong-Guang Xu and Min Yang the research method selection and technical support. All authors read and approved the final manuscript.

\section{Funding}

This study was supported by a grant from National Natural Science Foundation of China (82002322), Funding of "Peak" Training Program and "Panfeng" Innovation Team Project for Scientific Research of Yijishan Hospital, Wannan Medical College (grant no. GF2019G04, PF2019005, GF2019T02 and PF2019007) and Young and Middle-aged Key Project of Wannan Medical College(WK2020ZF16).

\section{Availability of data and materials}


The datasets used and/or analyzed in the current study are available from the corresponding author upon reasonable request.

\section{Ethics approval and consent to participate}

All animal procedures were approved under the guidelines of the Ethics Committee of the the First Affiliated Hospital of Wannan Medical College, Yijishan Hospital(Approval No. LLSC-2020-082).

\section{Consent for publication}

Not applicable.

\section{Competing interests}

The authors declare that they have no competing interests.

\section{Author details}

1 Department of Trauma Orthopedics, The First Affiliated Hospital of Wannan Medical College, Yijishan Hospital, No. 2, Zhe shan Xi Road, Wuhu 241001, Anhui, People's Republic of China. \2 Department of Spinal orthopedics, The First Affiliated Hospital of Wannan Medical College, Yijishan Hospital, No. 2, Zhe shan Xi Road, Wuhu 241001, Anhui, People's Republic of China.

\section{References}

1. Cosman F. Anabolic Therapy and Optimal Treatment Sequences for Patients With Osteoporosis at High Risk for Fracture. Endocrine practice: official journal of the American College of Endocrinology and the American Association of Clinical Endocrinologists. 2020;26(7):777 - 86. doi:10.4158/ep2019-0596.

2. Abidin N, Mitra S. Total vs. Bioavailable: Determining a Better $25(\mathrm{OH}) \mathrm{D}$ Index in Association with Bone Density and Muscle Mass in Postmenopausal Women. Metabolites. 2020;11(1). doi:10.3390/metabo11010023.

3. Tao Z, Zhou W, Wu X, Wang L, Yang M, Xie J, et al. Single-dose local administration of parathyroid hormone $(1-34, \mathrm{PTH})$ with $\beta$-tricalcium phosphate/collagen ( $\beta$-TCP/COL) enhances bone defect healing in ovariectomized rats. J Bone Miner Metab. 2019;37(1):28-35. doi:10.1007/s00774-0180906-3.

4. Liu X, Chin J, Qu X, Bi H, Liu Y, Yu Z, et al. The Beneficial Effect of Praeruptorin C on Osteoporotic Bone in Ovariectomized Mice via Suppression of Osteoclast Formation and Bone Resorption. Front Pharmacol. 2017;8:627. doi:10.3389/fphar.2017.00627.

5. Liang B, Shi Q, Xu J, Chai Y, Xu J. Poly (Glycerol Sebacate)-Based Bio-Artificial Multiporous Matrix for Bone Regeneration. Frontiers in chemistry. 2020;8:603577. doi:10.3389/fchem.2020.603577. 
6. Schmidt A. Autologous bone graft: Is it still the gold standard? Injury. 2021. doi:10.1016/j.injury.2021.01.043.

7. Bharadwaz A, Jayasuriya A. Osteogenic differentiation cues of the bone morphogenetic protein-9 (BMP-9) and its recent advances in bone tissue regeneration. Materials science \& engineering C, Materials for biological applications. 2021;120:111748. doi:10.1016/j.msec.2020.111748.

8. Matias M, Gomes V, Romao-Veiga M, Ribeiro V, Nunes P, Romagnoli G, et al. Silibinin Downregulates the NF-KB Pathway and NLRP1/NLRP3 Inflammasomes in Monocytes from Pregnant Women with Preeclampsia. Molecules. 2019;24(8). doi:10.3390/molecules24081548.

9. Federico A, Dallio M, Loguercio C. Silymarin/Silybin and Chronic Liver Disease: A Marriage of Many Years. Molecules. 2017;22(2). doi:10.3390/molecules22020191.

10. Shen Y, Zhao H, Wang Z, Guan W, Kang X, Tai X, et al. Silibinin declines blue light-induced apoptosis and inflammation through MEK/ERK/CREB of retinal ganglion cells. Artificial cells nanomedicine biotechnology. 2019;47(1):4059-65. doi:10.1080/21691401.2019.1671430.

11. Rajalakshmi S, Vimalraj S, Saravanan S, Raj Preeth D, Shairam M, Anuradha D. Synthesis and characterization of silibinin/phenanthroline/neocuproine copper(II) complexes for augmenting bone tissue regeneration: an in vitro analysis. Journal of biological inorganic chemistry: JBIC : a publication of the Society of Biological Inorganic Chemistry. 2018;23(5):753-62. doi:10.1007/s00775-018-1566-4.

12. Vimalraj S, Rajalakshmi S, Saravanan S, Raj Preeth D, LA Vasanthi R, Shairam M, et al. Synthesis and characterization of zinc-silibinin complexes: A potential bioactive compound with angiogenic, and antibacterial activity for bone tissue engineering. Colloids surfaces B Biointerfaces. 2018;167:13443. doi:10.1016/j.colsurfb.2018.04.007.

13. Ying $X$, Sun $L$, Chen $X, X u H$, Guo X, Chen $H$, et al. Silibinin promotes osteoblast differentiation of human bone marrow stromal cells via bone morphogenetic protein signaling. Eur J Pharmacol. 2013;721:225-30. doi:10.1016/j.ejphar.2013.09.031.

14. Wang T, Cai L, Wang Y, Wang Q, Lu D, Chen H, et al. The protective effects of silibinin in the treatment of streptozotocin-induced diabetic osteoporosis in rats. Biomedicine pharmacotherapy = Biomedecine pharmacotherapie. 2017;89:681-8. doi:10.1016/j.biopha.2017.02.018.

15. Ying $X$, Chen $X$, Liu H, Nie P, Shui $X$, Shen $Y$, et al. Silibinin alleviates high glucose-suppressed osteogenic differentiation of human bone marrow stromal cells via antioxidant effect and PI3K/Akt signaling. Eur J Pharmacol. 2015;765:394-401. doi:10.1016/j.ejphar.2015.09.005.

16. Wong P, Tan T, Chan C, Laxton V, Chan Y, Liu T, et al. The Role of Connexins in Wound Healing and Repair: Novel Therapeutic Approaches. Frontiers in physiology. 2016;7:596. doi:10.3389/fphys.2016.00596.

17. Xu H, Abuduwufuer A, Lv W, Zhou Z, Yang Y, Zhang C, et al. The role of HIF-1a-VEGF pathway in bronchiolitis obliterans after lung transplantation. J Cardiothorac Surg. 2019;14(1):27. doi:10.1186/s13019-019-0832-z. 
18. Xiao S, Zhang D, Liu Z, Jin W, Huang G, Wei Z, et al. Diabetes-induced glucolipotoxicity impairs wound healing ability of adipose-derived stem cells-through the miR-1248/CITED2/HIF-1 a pathway. Aging. 2020;12(8):6947-65. doi:10.18632/aging.103053.

19. Semenova D, Bogdanova M, Kostina A, Golovkin A, Kostareva A, Malashicheva A. Dose-dependent mechanism of Notch action in promoting osteogenic differentiation of mesenchymal stem cells. Cell tissue research. 2020;379(1):169-79. doi:10.1007/s00441-019-03130-7.

20. Tao ZS, Lv YX, Cui W, Huang ZL, Tu KK, Zhou Q, et al. Effect of teriparatide on repair of femoral metaphyseal defect in ovariectomized rats. Z Gerontol Geriatr. 2016;49(5):423-8. doi:10.1007/s00391-015-0949-1.

21. Tao ZS, Wu XJ, Zhou WS, Wu XJ, Liao W, Yang M, et al. Local administration of aspirin with betatricalcium phosphate/poly-lactic-co-glycolic acid (beta-TCP/PLGA) could enhance osteoporotic bone regeneration. J Bone Miner Metab. 2019;37(6):1026-35. doi:10.1007/s00774-019-01008-w.

22. Tao ZS, Zhou WS, Xu HG, Yang M. Aspirin modified strontium-doped beta-tricalcium phosphate can accelerate the healing of femoral metaphyseal defects in ovariectomized rats. Biomed Pharmacother. 2020;132:110911. doi:10.1016/j.biopha.2020.110911.

23. Tao ZS, Zhou WS, Wu XJ, Wang L, Yang M, Xie JB, et al. Single-dose local administration of parathyroid hormone (1-34, PTH) with beta-tricalcium phosphate/collagen (beta-TCP/COL) enhances bone defect healing in ovariectomized rats. J Bone Miner Metab. 2019;37(1):28-35. doi:10.1007/s00774-018-0906-3.

24. Alsaggar M, Bdour S, Ababneh Q, El-Elimat T, Qinna N, Alzoubi K. Silibinin attenuates adipose tissue inflammation and reverses obesity and its complications in diet-induced obesity model in mice. BMC pharmacology toxicology. 2020;21(1):8. doi:10.1186/s40360-020-0385-8.

25. Li YF, Li XD, Bao CY, Chen QM, Zhang H, Hu J. Promotion of peri-implant bone healing by systemically administered parathyroid hormone (1-34) and zoledronic acid adsorbed onto the implant surface. Osteoporosis international: a journal established as result of cooperation between the European Foundation for Osteoporosis and the National Osteoporosis Foundation of the USA. 2013;24(3):1063-71. doi:10.1007/s00198-012-2258-5.

26. Gabet Y, Kohavi D, Kohler T, Baras M, Müller R, Bab I. Trabecular bone gradient in rat long bone metaphyses: mathematical modeling and application to morphometric measurements and correction of implant positioning. J Bone Miner Res. 2008;23(1):48-57.

27. Hao Y, Yingjie H, Zhang G, Ge Z, Wang Y, Yisheng W, et al. Changes of microstructure and mineralized tissue in the middle and late phase of osteoporotic fracture healing in rats. Bone. 2007;41(4):631-8. doi:10.1016/j.bone.2007.06.006.

28. He Y, Zhang G, Pan X, Liu Z, Zheng L, Chan C, et al. Impaired bone healing pattern in mice with ovariectomy-induced osteoporosis: A drill-hole defect model. Bone. 2011;48(6):1388-400. doi:10.1016/j.bone.2011.03.720.

29. Luo Z, Shang X, Zhang H, Wang G, Massey P, Barton S, et al. Notch Signaling in Osteogenesis, Osteoclastogenesis, and Angiogenesis. Am J Pathol. 2019;189(8):1495-500. 
doi:10.1016/j.ajpath.2019.05.005.

30. Liao J, Wei Q, Zou Y, Fan J, Song D, Cui J, et al. Notch Signaling Augments BMP9-Induced Bone Formation by Promoting the Osteogenesis-Angiogenesis Coupling Process in Mesenchymal Stem Cells (MSCs). Cellular physiology and biochemistry: international journal of experimental cellular physiology, biochemistry, and pharmacology. 2017;41(5):1905-23. doi:10.1159/000471945.

31. Jiang L, Sheng K, Wang C, Xue D, Pan Z. The Effect of MMP-2 Inhibitor 1 on Osteogenesis and Angiogenesis During Bone Regeneration. Frontiers in cell developmental biology. 2020;8:596783. doi:10.3389/fcell.2020.596783.

32. Chen L, Zhang R, Xie J, Yang J, Fang K, Hong C, et al. STAT3 activation by catalpol promotes osteogenesis-angiogenesis coupling, thus accelerating osteoporotic bone repair. Stem Cell Res Ther. 2021;12(1):108. doi:10.1186/s13287-021-02178-z.

33. Zou D, Han W, You S, Ye D, Wang L, Wang S, et al. In vitro study of enhanced osteogenesis induced by HIF-1a-transduced bone marrow stem cells. Cell proliferation. 2011;44(3):234-43. doi:10.1111/j.1365-2184.2011.00747.x.

34. Sharma S, Xue Y, Xing Z, Yassin M, Sun Y, Lorens J, et al. Adenoviral mediated mono delivery of BMP2 is superior to the combined delivery of BMP2 and VEGFA in bone regeneration in a criticalsized rat calvarial bone defect. Bone reports. 2019;10:100205. doi:10.1016/j.bonr.2019.100205.

35. Poudel S, Min C, Lee J, Shin Y, Kwon T, Jeon Y, et al. Local supplementation with plant-derived recombinant human FGF2 protein enhances bone formation in critical-sized calvarial defects. J Bone Miner Metab. 2019;37(5):900-12. doi:10.1007/s00774-019-00993-2.

36. Guo Q, Yang J, Chen Y, Jin X, Li Z, Wen X, et al. Salidroside improves angiogenesis-osteogenesis coupling by regulating the HIF-1a/VEGF signalling pathway in the bone environment. Eur $\mathrm{J}$ Pharmacol. 2020;884:173394. doi:10.1016/j.ejphar.2020.173394.

\section{Figures}




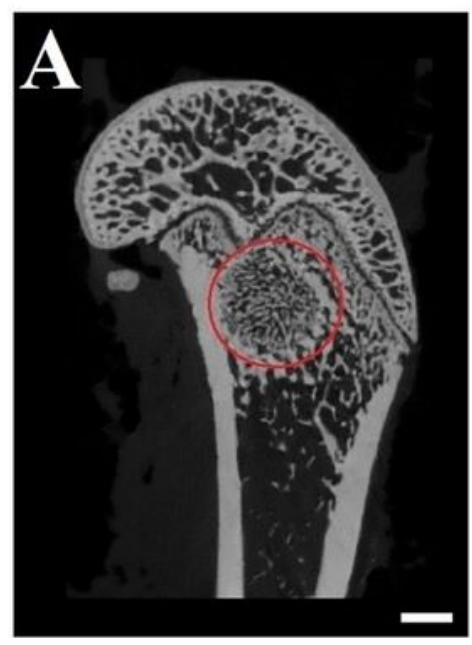

a
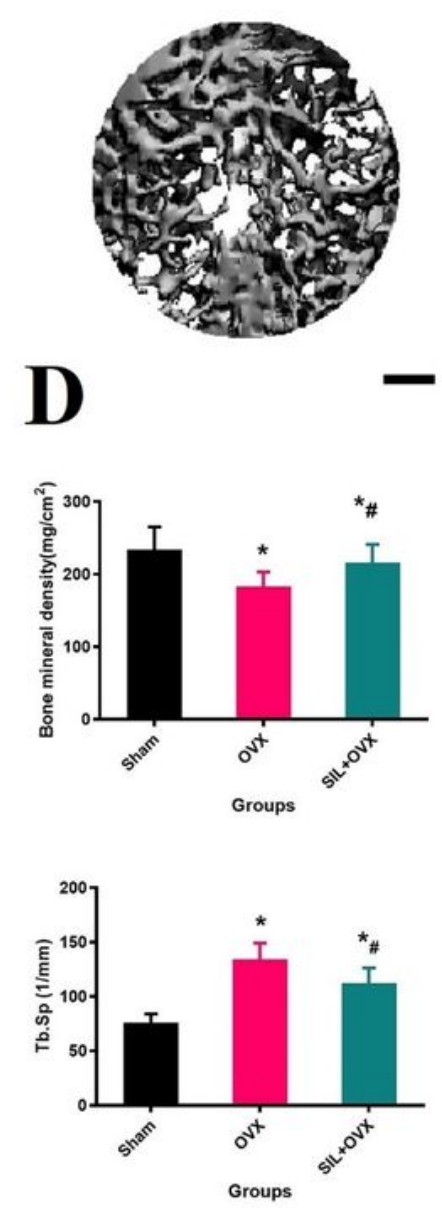

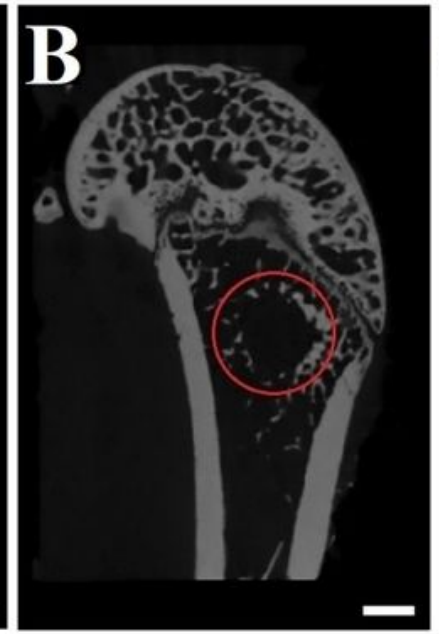

b
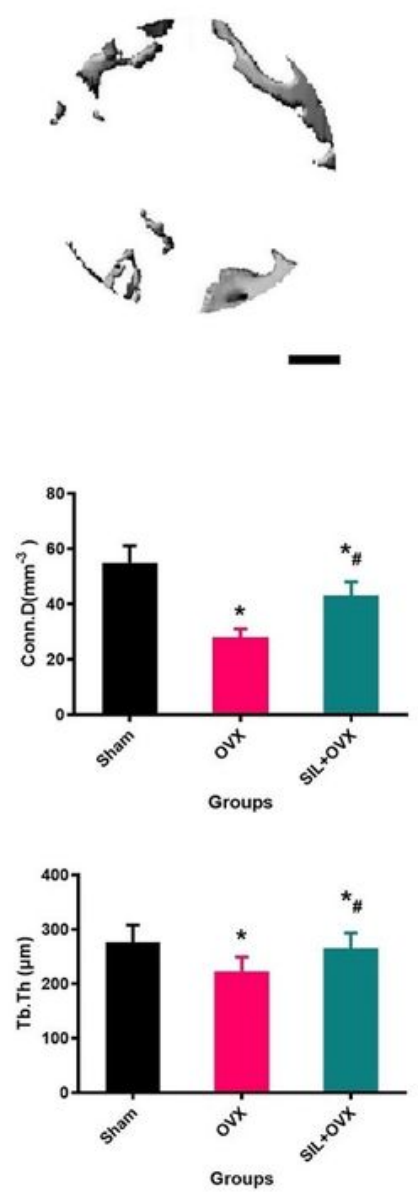

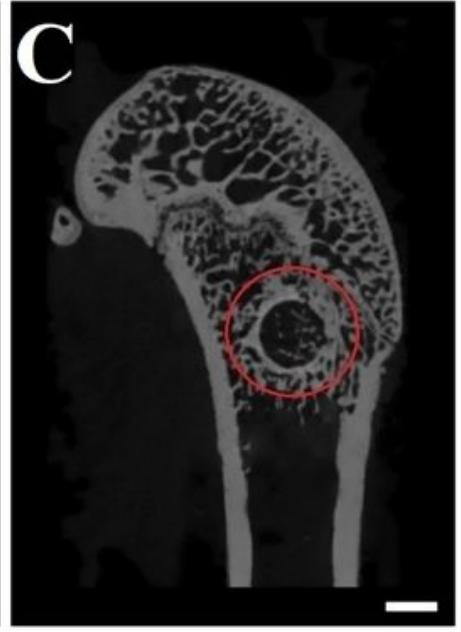

C
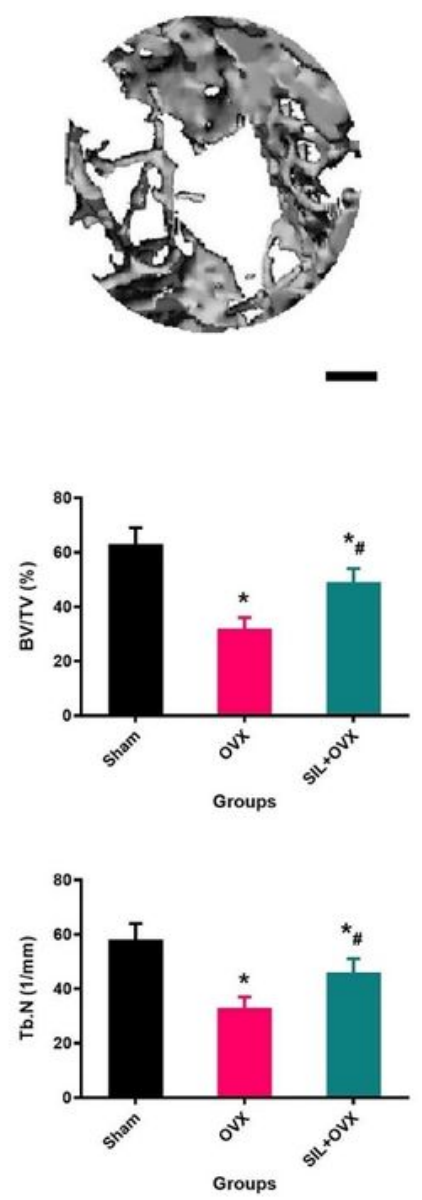

\section{Figure 1}

Micro-CT images of the distal femur 12 weeks after biomaterials implanted from group of Sham $(A, a)$, OVX (B, b) and SIL+OVX (C, c); The scale bar represents $2 \mathrm{~mm}$. D: Quantitative results of new trabeculae bone in defect area including BMD, BV/TV, Tb. N, Conn.D, Tb. Th, and Tb.Sp. *Vs. Sham group, $p<0.05$, $\#$ Vs. OVX, $\mathrm{p}<0.05$. 
A

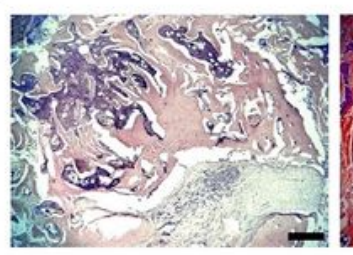

Sham

B

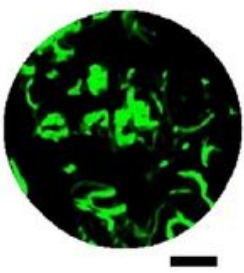

Sham

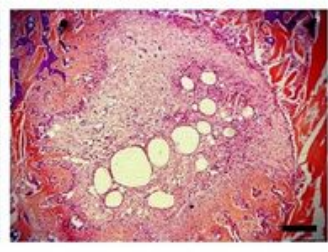

OVX

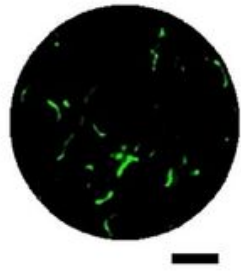

OVX

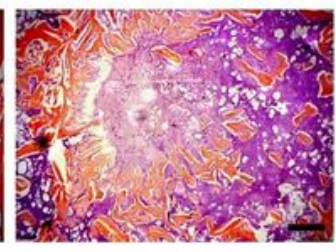

SIL+OVX

C
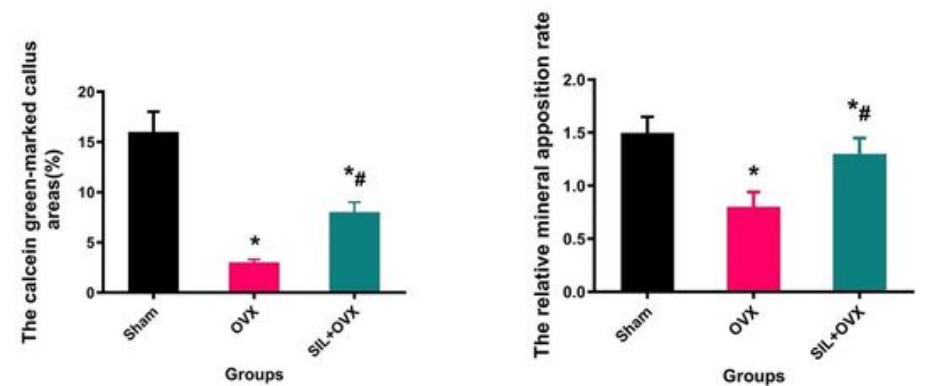

D
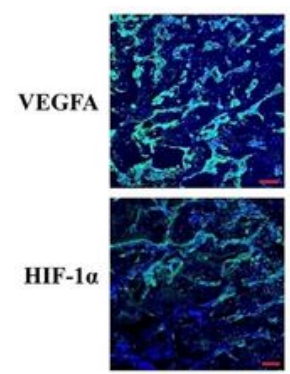

Sham

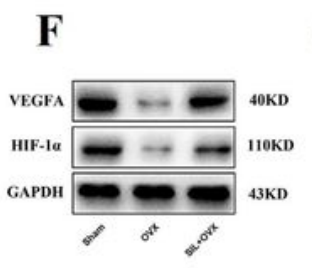

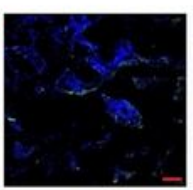

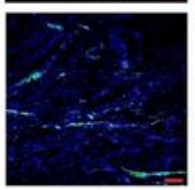

ovx
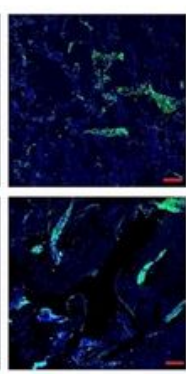

SIL+OVX

E

G

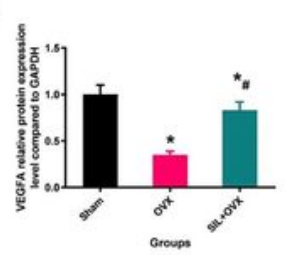

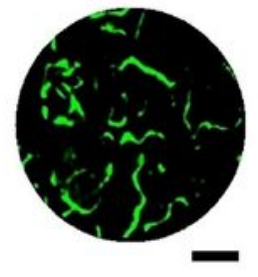

SIL+OVX
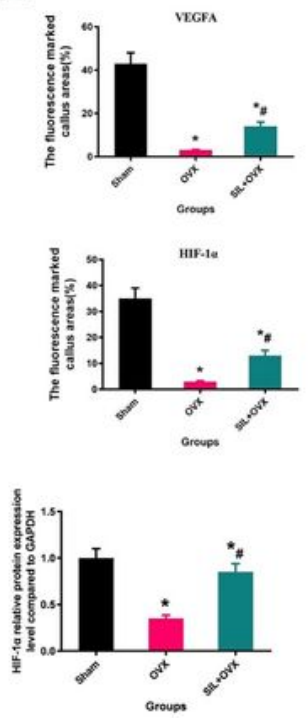

\section{Figure 2}

Bone regeneration of defected area by histological $(A$, magnification, $\times 10)$ and fluorescent analysis $(B$, magnification, $\times 200)$. C. Total fluorescently marked defect area(\%) and Relative bone mineralization (green/green marked defect area) after SIL treatment. Bone tissue protein expression of HIF-1a and VEGFA in defect area measured by immunofluorescence(D) and WB(F); The quantitative results were 
expressed as fluorescently marked defect area(E) and relative protein expression(G). *Vs. Sham group, $p<0.05$, \#Vs. OVX, p<0.05.
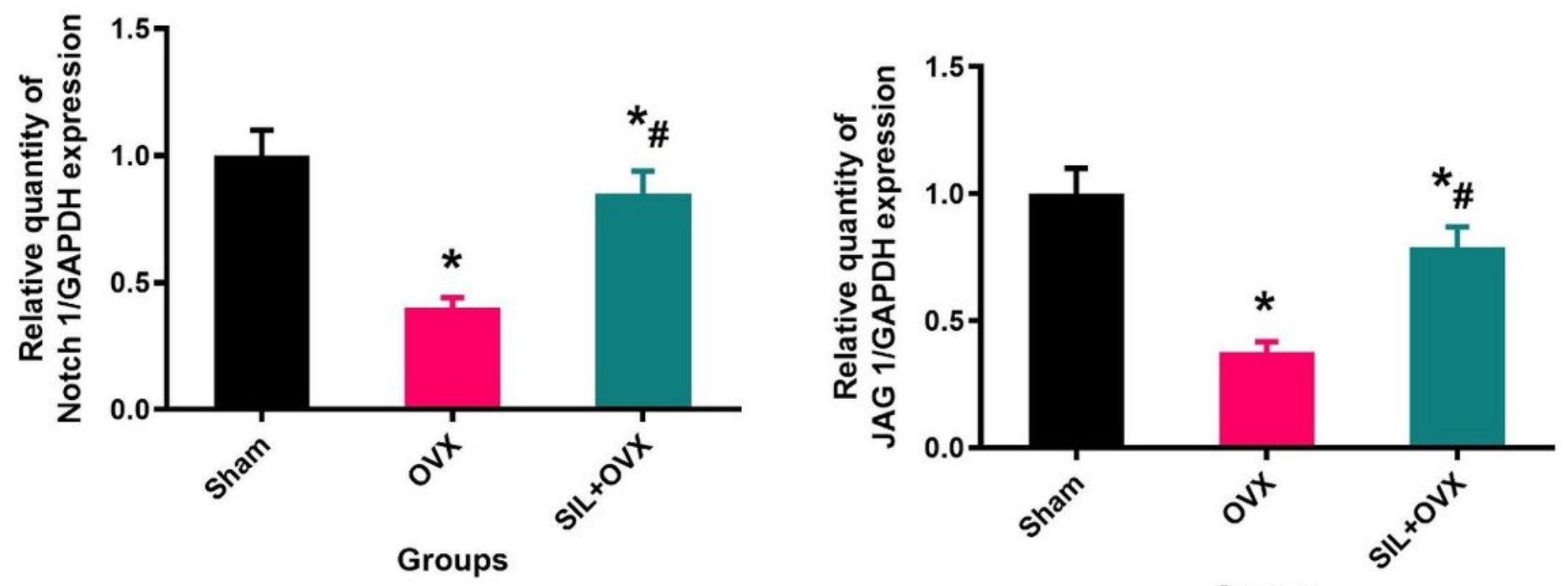

Groups
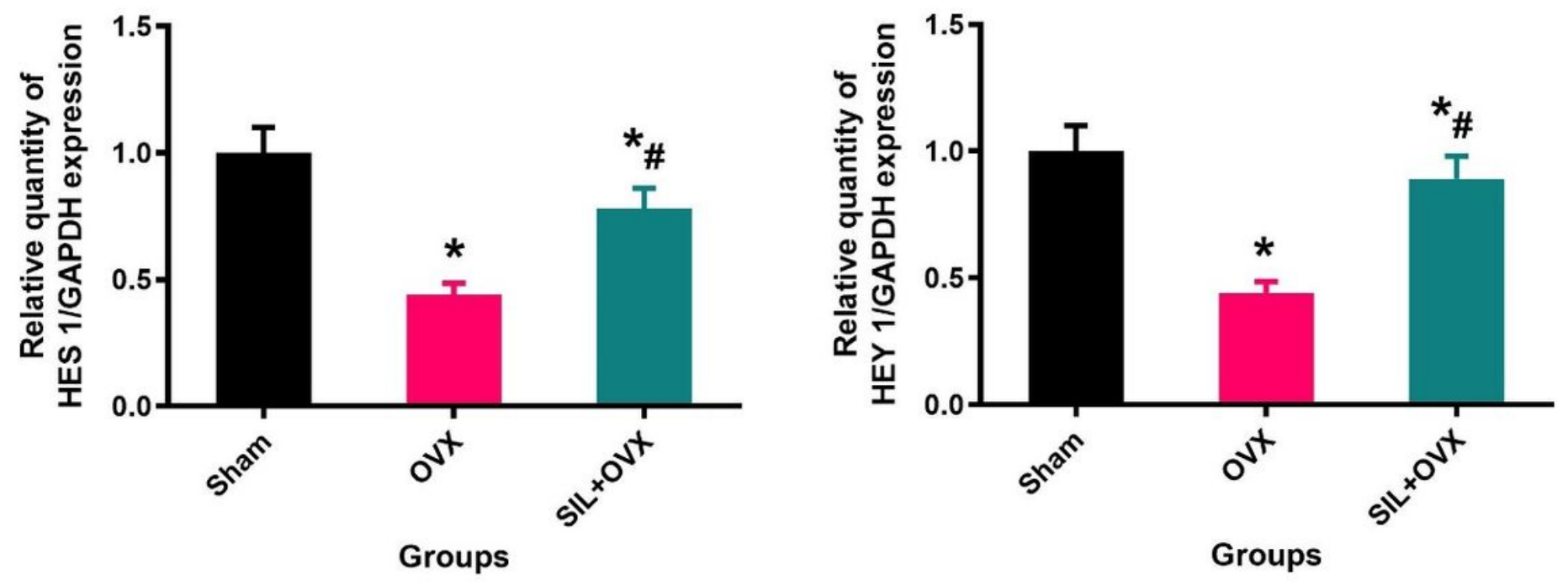

Figure 3

Gene expression of defect area bone tissue after different treatment. *Vs. Sham group, $p<0.05, \# V s$. OVX, $\mathrm{p}<0.05$. 
A

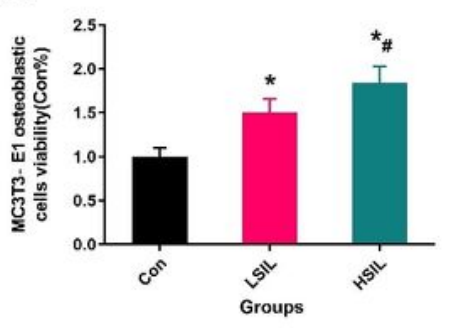

D

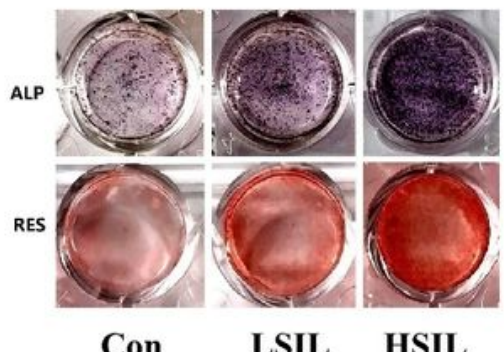

G

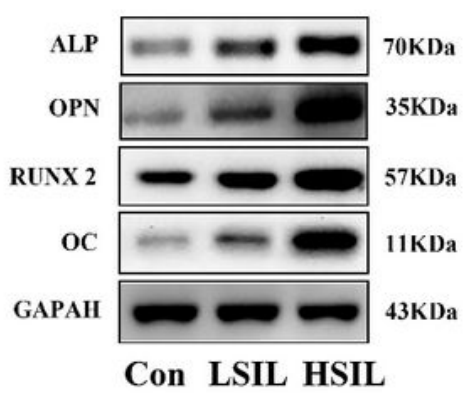

Con LSIL HSIL

$$
\text { I }
$$

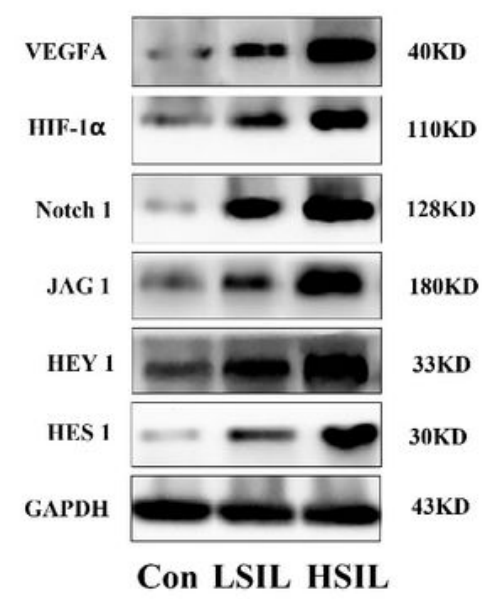

B

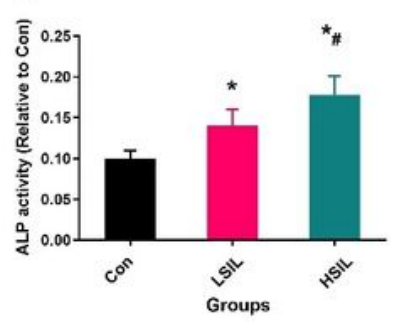

E

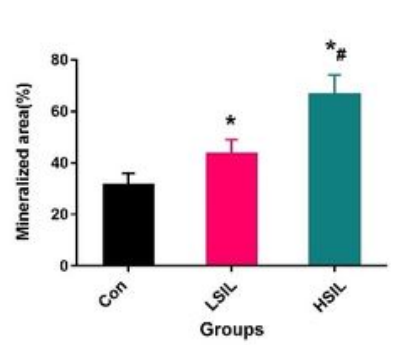

C

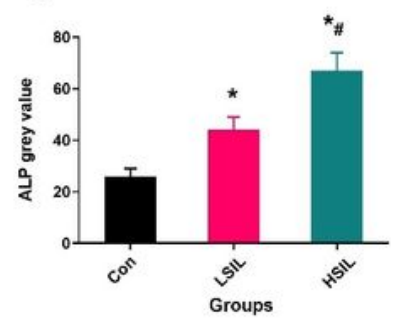

F

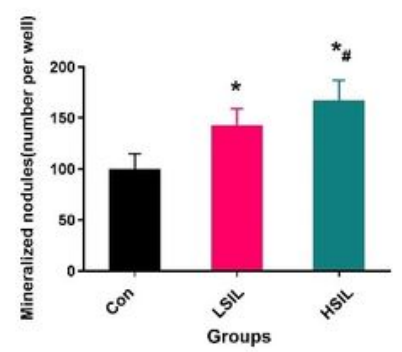

H

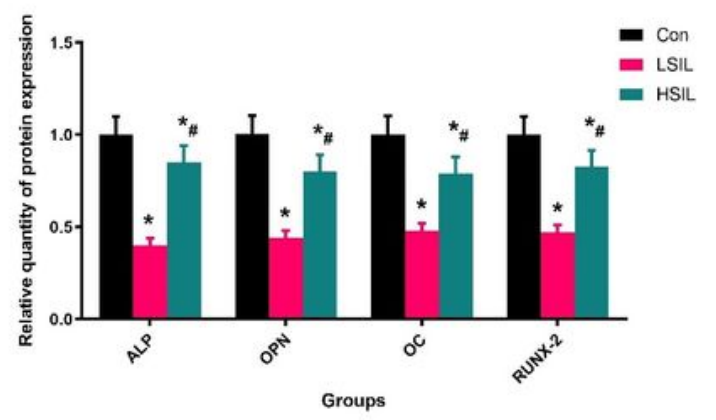

J

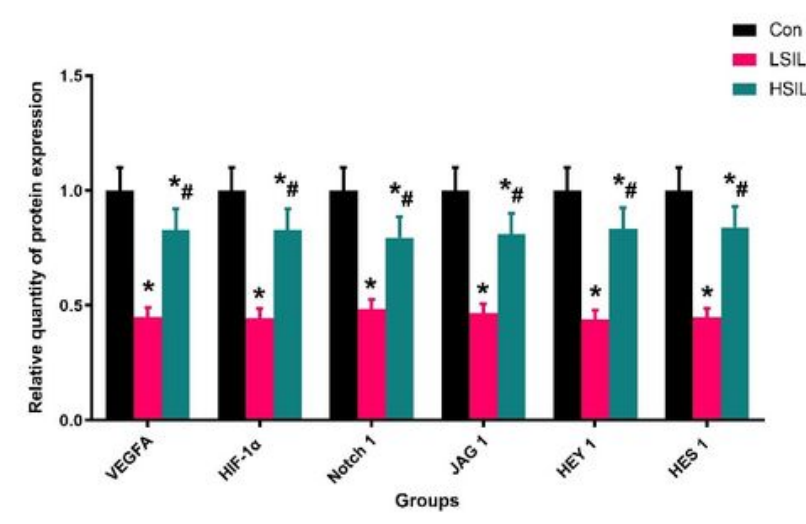

- Con

m LSIL

Figure 4

Different treatments affect cell activity(A) and function(D) and related protein expression(G). The quantification of mineralized nodules $(E)$, mineralized area $(F)$, ALP activity $(B)$, ALP gray value(C) and related protein expression(G-J). *Vs. Con group, $p<0.05$, \#Vs. LSIL, $p<0.05$. 